\title{
An Integrated Fixed/Mobile Network for 3G Services
}

\author{
J. Charles Francis ${ }^{1}$ \\ ${ }^{1}$ Swisscom, Corporate Technology, CH-3000 Bern 29, Switzerland \\ JohnCharles.Francis@Swisscom.com
}

\begin{abstract}
Today, there are separate networks infrastructures for fixed and mobile communications, and for Internet access support. In future, however, a layered architecture with diverse service provision based on common network technologies is foreseen. The network evolution towards this goal is considered in the context of the standardisation work for UMTS. The scope of this paper includes 3GPP work for Release 2000 and wireline access to $3^{\text {rd }}$ Generation mobile services.
\end{abstract}

\section{Introduction}

Traditionally, there have been separate networks infrastructures for fixed and mobile communications, and for Internet access support. In the EURESCOM P919 project, however, an alternative approach has been investigated using a layered architecture with diverse service provision based on common network technologies (Figure 1). As part of this study, Multi Protocol Label Switching (MPLS) technology has been investigated as a possible means to support seamless service provision across diverse access media, with potential cost savings in network operation and infrastructure.

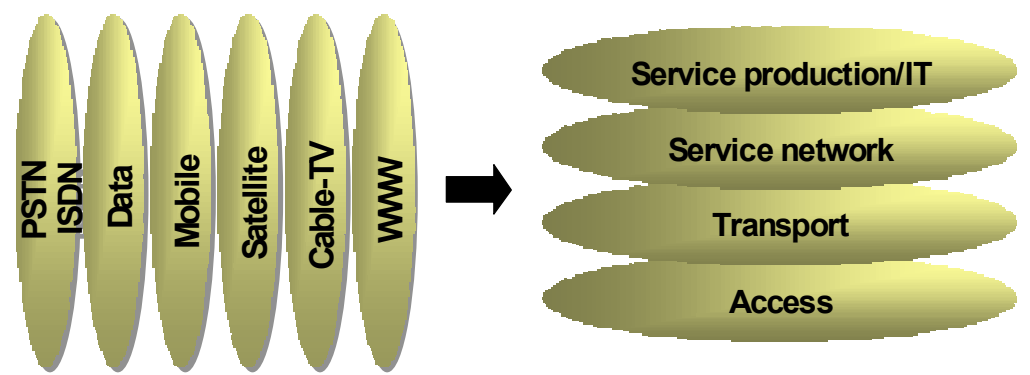

Fig. 1. Shift from dedicated service networks towards a layered architecture with common network technologies 


\section{Evolution of GSM towards $3^{\text {rd }}$ Generation Capabilities}

The cellular system deployed by European mobile operators is based on GSM technology and operates in the $900 \mathrm{MHz}$ and $1800 \mathrm{MHz}$ frequency bands. Work within the ETSI Special Mobile Group (SMG) has specified an evolved radio technology, the Enhanced Data rates for GSM Evolution (EDGE), which uses the existing GSM frequency bands to achieve bitrates of up to $384 \mathrm{kBit} / \mathrm{s}$. The radio access for UMTS, the so-called UMTS Terrestrial Radio Access Network (UTRAN) operates in a higher frequency range $(2 \mathrm{GHz})$ and achieves bitrates of up to $2 \mathrm{MBit} / \mathrm{s}$. The same core network standards are defined for both EDGE and UTRAN, which will ensure compatible services. In this context, specification work for the future mobile core network is carried out by the $3^{\text {rd }}$ Generation Partnership Project (3GPP).

When UMTS systems based on the so-called 3GPP Release ' 99 specifications are deployed by GSM operators in 2002, it is highly probable that the UTRAN will be interconnected with the existing GSM and General Packet Radio Service (GPRS) core networks (Figure 2). The initial deployment of UMTS may well be limited to isolated islands (e.g., city centres, business areas, industrial plants) and in such a case, the GSM infrastructure will provide limited service support between UMTS islands. To allow the UTRAN to communicate with the GSM and GPRS networks, so-called Interworking Units are needed. The interface between the UTRAN and the core network is the $I u$-interface and is based on ATM.

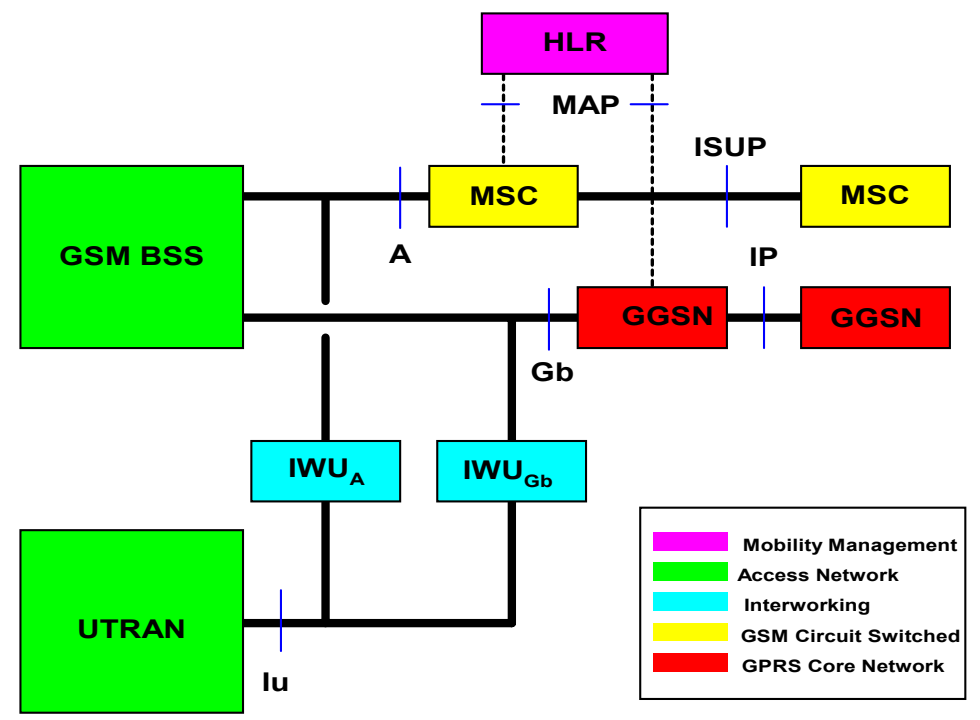

Fig. 2. Interconnection of a UMTS Terrestrial Radio Access Network (UTRAN) with the existing (legacy) GSM/GPRS Core Network 


\section{The 3GPP Release 2000}

At the beginning of the year, work started within 3GPP on Release 2000. This specification will include functional enhancements to Release ' 99 and transportindependent support of UMTS with an "all-IP" option. Below are some of the 3GPP architectural principles for Release 2000.

Transport Independence (to control heterogeneous bearer mechanisms): The GSM/UMTS Core Network architecture will be independent of the underlying transport mechanism (e.g. STM, ATM or IP). Furthermore, operators will have the freedom to utilise a single or any combination of transport technologies.

Standardised alternatives for transport mechanisms: Alternatives for the signalling transport (e.g. SS7, SIGTRAN) for the service control, call control and bearer control protocols as well as the alternatives for the user plane transport will be standardised for relevant transport mechanisms.

Decomposition of network functions: The GSM/UMTS reference architecture allIP option will be defined in terms of separate functions and clear interfaces such that it is possible to separate transport from signalling. This has the objective of separation of call/session, mobility and service control. This is intended to give operators the freedom to provision, dimension and upgrade these network functions in a modular fashion, providing flexibility and scalability of network implementations.

Flexible traffic processing function placement: The GSM/UMTS reference architecture will allow operators to place the traffic processing functions in the most practical, cost-effective part of the network.

Use of Internet protocols: The GSM/UMTS reference architecture will use, as appropriate, existing/evolving Internet protocols, e.g., to support multimedia services, interoperability with other next generation fixed or mobile networks, and media gateway controllers.

Support for a variety of mobile equipment: The GSM/UMTS reference architecture will support a range of different terminal types (simple speech only terminals, multimedia terminals, PDAs, Laptops, etc.). One particular aspect is that not all terminals may be able to support end-to-end IP capabilities.

Independence of access technology: The GSM/UMTS reference architecture will be designed to ensure that a common core network can be used with multiple wireless and wireline access technologies (e.g., xDSL, Cable, Wireless LAN, Digital Broadcast, all IMT2000 radio access technologies).

Support for roaming onto other $2 G$ and $3 G$ mobile networks: The GSM/UMTS reference architecture will be designed to facilitate roaming between different network types.

Support of Service Requirements: The GSM/UMTS reference architecture will include mechanisms for operators and third-parties to rapidly develop and provide services and for users to customise their service profile

Support of regulatory requirements: The GSM/UMTS reference architecture will include features to support regulatory requirements such as legal intercept, number portability and other regional requirements.

Separation between Bearer level, Call control level and Service level:

- Use of different access technology to connect the "IP multimedia core network subsystem": The IP multimedia domain will be connected to the 
bearer network at a fixed reference point (anchor point), thus hiding the micro mobility of the user equipment (terminal), but it will not hide roaming. This reference point will be independent of the access technology which can be GPRS, UMTS packet-switched or any relevant wireless, wired-line access technology as long as they provide transport of user packets up to this reference point and hide the micro-mobility of the User Equipment. As a consequence, the behaviour of the multimedia call control server can be the same whatever the access technology (radio or wired-line).

- The access to the IP multimedia core network subsystem will be supported by the packet-switched domain at the, so-called, Gi interface: The packetswitched domain provides bearers that will be used by the user equipment for its signalling and to provide user plane exchanges with multimedia (H323/SIP) call control servers and gateways. These servers / gateways will be located behind the GGSN, acting as an anchor point for the mobility, implying that when the terminal is moving, the call control server is not changed as long as the user equipment (terminal) is registered on this server.

The specifications need to support both circuit-mode and packet-mode domains: Considering the traffic mix resulting from the set of $3 \mathrm{G}$ services and the need for flexible evolution paths, it will be necessary to have separate circuit-switched and packet-switched domains. Each domain will handle its own signalling traffic, switching and routing.

Keep network functions separate from radio access functions: The same network should support a variety of access choices, and access technologies may evolve further. Therefore, network functions such as call control, service control, etc. should remain separate from access functions and ideally should be independent of the choice of access. This implies that the same core network should be able to interface to a variety of radio access networks.

\section{Wireline and Cordless Access to $3^{\text {rd }}$ Generation Mobile Services}

From the perspective of an integrated fixed/mobile core network, wired and wireless access are just different ways of accessing the same services. xDLS modems can be introduced to upgrade the capability of the copper local loop allowing a bandwidth of several Mbit/s. A UMTS, or other Iu-compliant, basestation can be added at the customer site to provide cordless access to $3 \mathrm{G}$ mobile services. An Interworking Unit (IWU) can be introduced at the $I u$-interface to separate traffic into the legacy circuitswitched and packet networks (Figure 3). This is analogous to the separation of UTRAN traffic into GSM circuit-switched and GPRS types, as shown in Figure 2. Direct connection to a UMTS core network can integrate connection-oriented and packet-based traffic (Figure 4). 


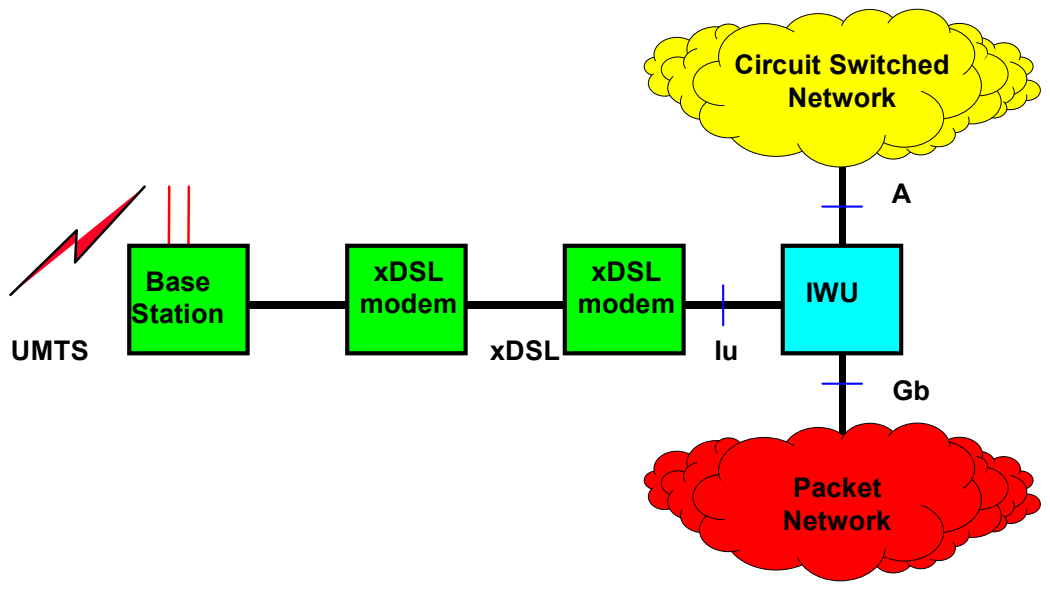

Fig. 3. Fixed network evolution towards UMTS. The copper local loop has been upgraded to xDSL and a UMTS basestation providing cordless access has been added at the customer premises. At the operator side, an Interworking Unit (IWU) separates circuit-switched traffic into respective (legacy) networks in a similar manner to Figure 2

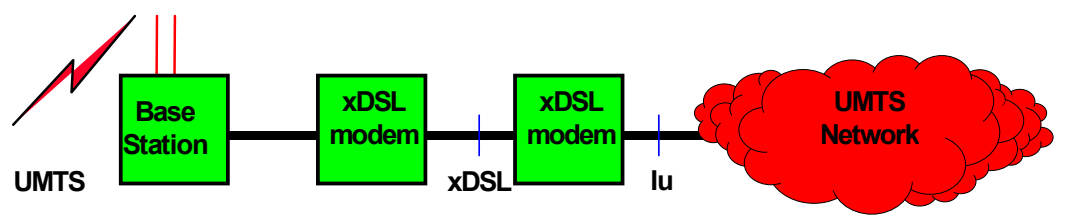

Fig. 4. In a future integrated scenario, xDSL can be connected to an integrated UMTS core network which handles both circuit-switched and packet traffic

\section{Protocol Considerations}

Over the next few years, telecommunication backbone networks will implement IPbased connectionless packet switching techniques supporting voice and multimedia traffic. Based on new QoS mechanisms and supported by centralised architectures, real time services will be enabled.

The evolution from the present circuit-switched networks to connectionless packetswitched networks will be made possible by the introduction of gateways. Gateways handle all the interworking functions, translating information between packet-based VoIP networks and circuit-switched networks. The entry gateway encodes the speech into a set of compressed voice frames, packetizes them into Real-Time Protocol 
packets and forwards them over IP or ATM core networks via the network interface. The gateways can be divided into three groups:

1. Media Gateways (MG) that handle the conversion of media streams from circuit format to packet format.

2. Media Gateway Controllers (MGC, Call Agents) that manage the packet network connections. In some configurations the $\mathrm{MGC}$ has integrated the Signalling Gateway function.

3. Signalling Gateways which constitute the interface to the circuit-switched networks with SS7 signalling.

For the communication between the MGCs (Call Agents) and the MGs, a media gateway call control protocol is used. Based on precursors MGCP and SGCP, the IETF MEGACO/ITU-T H.248 protocol is currently in the process of being standardised jointly by IETF and ITU-T. The evolution of the IP-network may be as follows:

1. In a first step, the CCS7 signalling trunks from the national/international telephony networks can be connected to the Media Gateway Controllers. The voice trunks are terminated at the Media Gateways. For the transport of the BICC protocol (between the Media Gateway Controllers) and the media streams (RTP between the Media Gateways), an ATM / MPLS Core Network can be used.

2. In a second step, the Media Gateways can be used for the connection of existing local exchanges. The LANs are directly connected to the MGs which are encoding the media into a set of compressed voice frames, packetizing them into RTP packets over IP. The CCS7 signalling from other National/International telephony networks can be connected to the Signalling Gateway Function (which may be collocated with the Media Gateway Controllers) using SCTP over IP.

3. In a third step, the IP (or another packet-switched network) can be connected over Multi Protocol Lambda Switching Network to DWDM. Other algorithms or Multi Protocols are under consideration.

Indicative solutions are shown in Figures 5 and 6. 


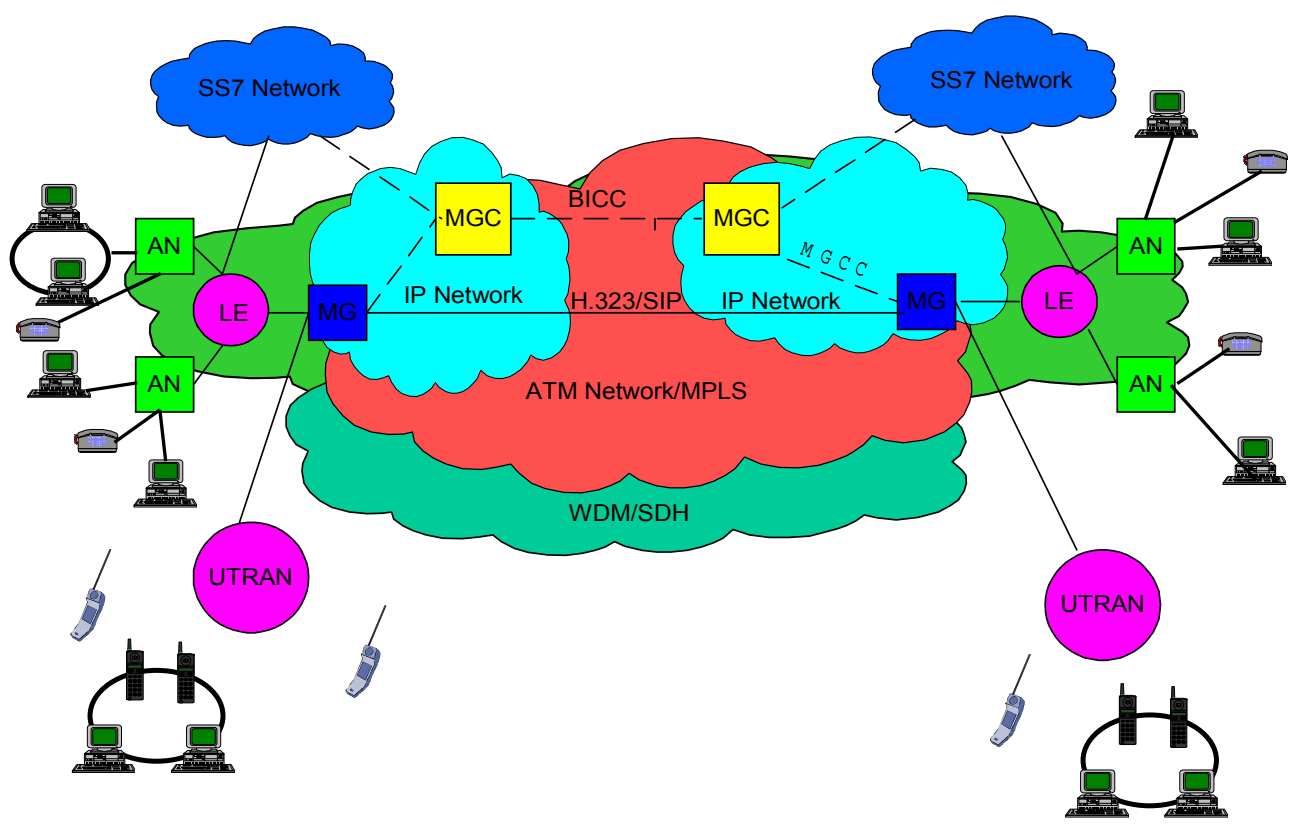

Fig. 5. Support of 3G mobile services by Multi Protocol Label Switching (MPLS)

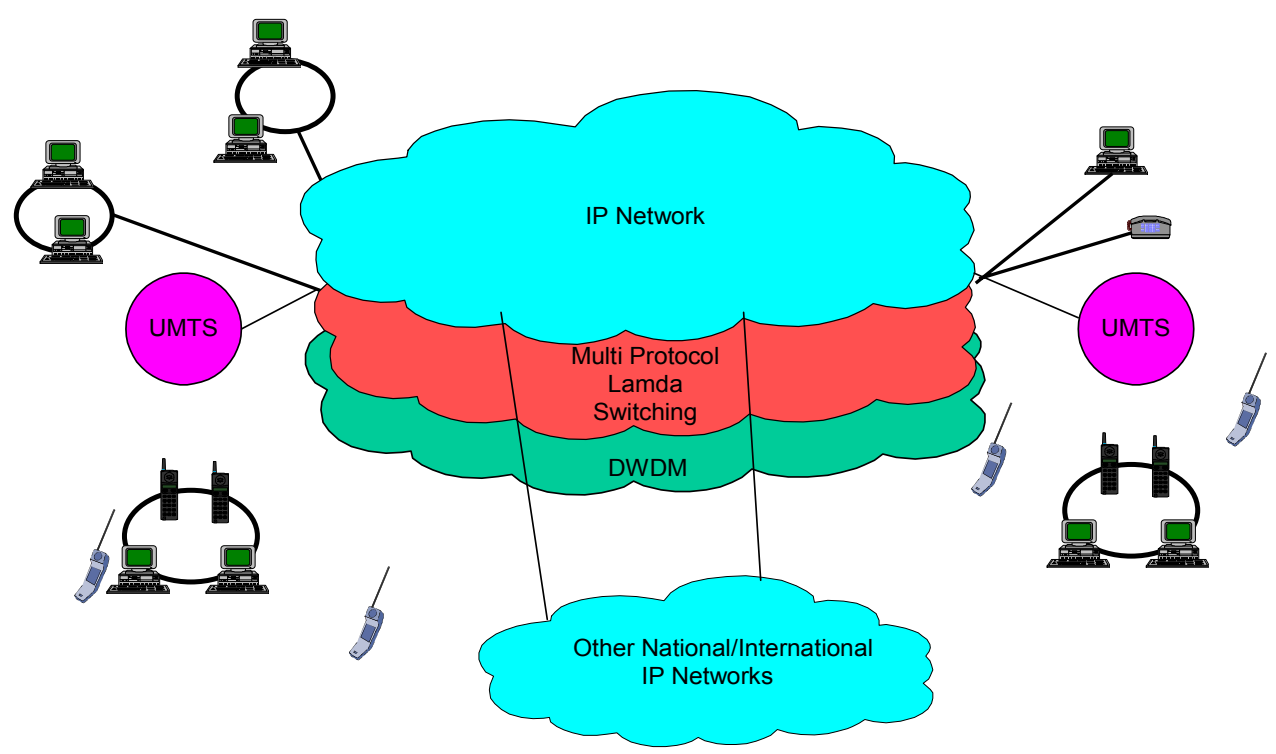

Fig. 6. An integrated fixed/mobile network for $3 \mathrm{G}$ services 


\section{Conclusions}

The technical work for the evolution of GSM towards UMTS is carried out by the $3^{\text {rd }}$ Generation Partnership Project (3GPP) and the currently approved specifications are referred to as Release '99. The specifications have been developed from a GSMoperator perspective and are aligned with the ETSI Special Mobile Group (SMG) work on GSM evolution based on EDGE radio technology. ETSI core network standards for $3^{\text {rd }}$ Generation Mobile are based 3GPP specifications and the 3GPP is work is therefore critical for both future GSM and UMTS. Mobile telecommunications in Europe is highly constrained by ETSI standards as appropriate international signalling for roaming users and common terminal standards must be supported. The specifications for Release ' 99 have been based on interworking to the legacy GSM/GPRS network. Current 3GPP work for Release 2000 is looking to transport-independent solutions including an all-IP option.

To achieve an integrated fixed/mobile network for $3 \mathrm{G}$ services, a primary issue is to bring wireline/cordless access into the $3^{\text {rd }}$ Generation mobile perspective. Options exist to upgrade the fixed network to provide a $3 \mathrm{G}$ cordless service and a variety of radio access technologies are available including UMTS, BLUETOOTH, BRAN, DECT and Wireless LAN. The same mobile services should be available independently of access media.

The use of wireline/cordless access to $3 \mathrm{G}$ mobile services will offer several benefits. For the operator, it reduces the demand on the cellular infrastructure and utilises existing investment in the local loop. For mobile subscribers, wired/cordless access to $3 \mathrm{G}$ mobile services offers a dedicated high-speed link which can provide access at higher bitrates than the UTRAN.

The future mobile services, whether based on UMTS or EDGE, will be multimedia. In the context of the new investment for these services, the opportunity will exist to add wired access / cordless access and achieve the goal of diverse service provision based on common network technologies.

\section{Abbreviations}

$\begin{array}{ll}\text { AN } & \text { Access Network } \\ \text { BICC } & \text { Bearer Independent Call Control } \\ \text { BSS } & \text { Base Station Subsystem } \\ \text { EDGE } & \text { Enhanced Data Rates for GSM Evolution } \\ \text { GGSN } & \text { Gateway GPRS Support Node } \\ \text { GPRS } & \text { General Packet Radio Service } \\ \text { HLR } & \text { Home Location Register } \\ \text { IWU } & \text { Inter Working Unit } \\ \text { LE } & \text { Local Exchange } \\ \text { MAP } & \text { Mobile Application Part } \\ \text { MG } & \text { Media Gateway } \\ \text { MGC } & \text { Media Gateway Controller } \\ \text { MGCP } & \text { Media Gateway Control Protocol }\end{array}$


MPLS

MSC

SGSN

SIP

UTRAN

WDM

xDSL
Multi-Protocol Label Switching

Mobile Switching Centre

Serving GPRS Support Node

Session Initiation Protocol

UMTS Terrestrial Radio Access Network

Wave Division Multiplexing

DSL (Digital Subscriber Line) technology

\section{Acknowledgement}

This paper reports work carried out within the EURESCOM P919 project in which the author has worked. The views expressed are those of the author and do not necessarily reflect the views of participating companies. 